\title{
Evaluation of electromagnetic fields on polluted polymer insulator under lightning impulse current
}

\begin{abstract}
This paper presents a simulation study of a polluted insulator under a direct lightning impulse current. The pollution condition was considered and divided into two cases which are partial and full pollution. Under those conditions, the electromagnetic profiles of the insulator were evaluated. In the study, six points of measurement recognised as the weak points of the insulator were introduced to be evaluated. Meanwhile the electric field and magnetic field values at the points were related to the common discharge and damage of polymer insulators. It was found that the pollution condition effectively influenced the electric field and magnetic field profile. A significant difference in the electric field value indicated at least $65.15 \%$ in both cases. For the magnetic field, at least a $10.9 \%$ percentage difference was recorded.

Keyword: Electromagnetic field; Polymer insulator; Lightning impulse current; Polluted insulator; Surface discharges
\end{abstract}

